\title{
A Collaborative Approach to Behavioral Health Care for Veterans and Their Families
}

M any veterans and their families struggle with behavioral health problems, yet they often face barriers to receiving adequate treatment. Notably, while many veterans are eligible to receive care at Department of Veterans Affairs (VA) facilities, family members are not and must seek care elsewhere. In seeking care in the community, veteran family members may face obstacles in ensuring that the care is sensitive to the issues that veteran families face and coordinated across providers.

Can a model of care that colocates and coordinates behavioral health services for veterans and their families address these barriers? The experiences in a new health center may shed light on this question. The Unified Behavioral Health Center (UBHC) for Military Veterans and Their Families is a public-private partnership between a VA medical center and a private-sector health care provider, both located in New York state. The center offers coordinated care for veterans and their families by locating VA and private providers side by side at the same facility.

RAND researchers evaluated the center's activities. The evaluation was intended to assess the viability of this new approach to behavioral health care, identify implementation challenges and successes, and assess the impact on patient health. The broader goal was to draw lessons to facilitate the center's replication elsewhere, should it prove successful.

The evaluation had two components. The first documented the center's capacity for delivering services (facilities, staff, technology, infrastructure) and the actual services delivered. The second assessed patient outcomes. For the first component, the evaluation used data from site visits and focus groups, as well as administrative data. For the second, it used patient-reported outcome data collected by UBHC staff.

\section{The Center Developed Capacity to Deliver a Range of Services}

- The UBHC is up and running and delivering a range of behavioral health services to veterans and their families (e.g., individual and group psychotherapy, family therapy, medication management).

- The center colocates and coordinates care across two independently governed sides. One side, the VA Clinic

\section{Key findings:}

- A partnership between the VA health system and a private-sector provider successfully implemented a behavioral health center that colocates and coordinates care for veterans and their families.

- Coordination of care for veterans and families showed promise in addressing barriers to care, and effective collaborative practices should be codified and institutionalized.

- Patients reported being happy with the services they received, and there is preliminary evidence that patients' symptoms and functioning improved significantly over time.

at Bay Shore, is operated by the VA and serves veterans; the other side, the Mildred and Frank Feinberg Division, is operated by Northwell Health System, a private-sector provider, and primarily serves the families of veterans. The two sides have two different managing authorities, procedures, and reporting requirements. Each side has separate entrances, information systems, and processes for monitoring performance.

- Coordination of care for family members receiving services on the different sides occurs primarily through team meetings, other in-person interactions, and phone contact among providers.

\section{The Center Overcame Key Start-Up Barriers, Although Other Challenges Remain}

- Gaining senior level buy-in from the local Veterans Affairs Medical Center (VAMC) and VA Central Office took time but was ultimately achieved by focusing on the potential benefits of the program for veterans and their families.

- Coordinating the construction of a new facility that met the needs of both Northwell Health and the Northport VAMC was challenging because of numerous regulatory considerations, some of which were not clear up front. 
- The funding model used in the first three years was not sustainable. The Feinberg Division initially provided services at no charge, generating no revenue, and relied on a grant and donations to pay operating expenses.

Recognizing this challenge, Northwell Health recently implemented a new billing system.

- VA Clinic at Bay Shore staff noted a lack of institutionalized and codified procedures for collaborative activities, which may present future challenges. Staff expressed a desire for clear directives that would support integration and collaboration and ensure that current effective strategies are preserved in the event of staffing changes.

\section{Staff Commitment and Strong Partnership Helped with Implementation}

- Staff at both Northwell Health and the Northport VAMC championed and facilitated the establishment of the UBHC. Since the center's establishment, staff within both systems have forged strong working relationships.

- A Robert Wood Johnson Foundation grant was a critical catalyst in establishing the center.

- Media attention helped to advertise the availability and services offered by the center.

\section{Service Use Differed for the Two Sides}

- The two sides of the center had different patterns of service use: The VA Clinic at Bay Shore provided fewer services to a larger number of individuals, while the Feinberg Division provided more-intensive services to a smaller number of individuals.

- Both sides, however, succeeded in becoming operational and delivering a substantial amount of services (more than 7,000 behavioral health encounters on each side of the center) in a relatively short time frame (three years). This was notable considering that it was a new center ramping up its capacity to provide care (e.g., through staffing) and reaching out to potential patients in the community for the first time.

\section{Patient Outcomes Improved}

- Adult patients treated on the Feinberg Division side of the UBHC showed improvement in key outcomes, including symptoms of depression (Figure 1) and posttraumatic stress disorder, family functioning, and quality of life. Child patients displayed fewer behavioral health problems (Figure 2). It is uncertain whether this effect is linked specifically to the care provided.

- UBHC patients consistently expressed satisfaction with their experiences at the center and the care they received. Patients reported several advantages of the family receiving coordinated care, and family members appreciated
Figure 1. Depressive Symptoms Decreased for Adult Patients

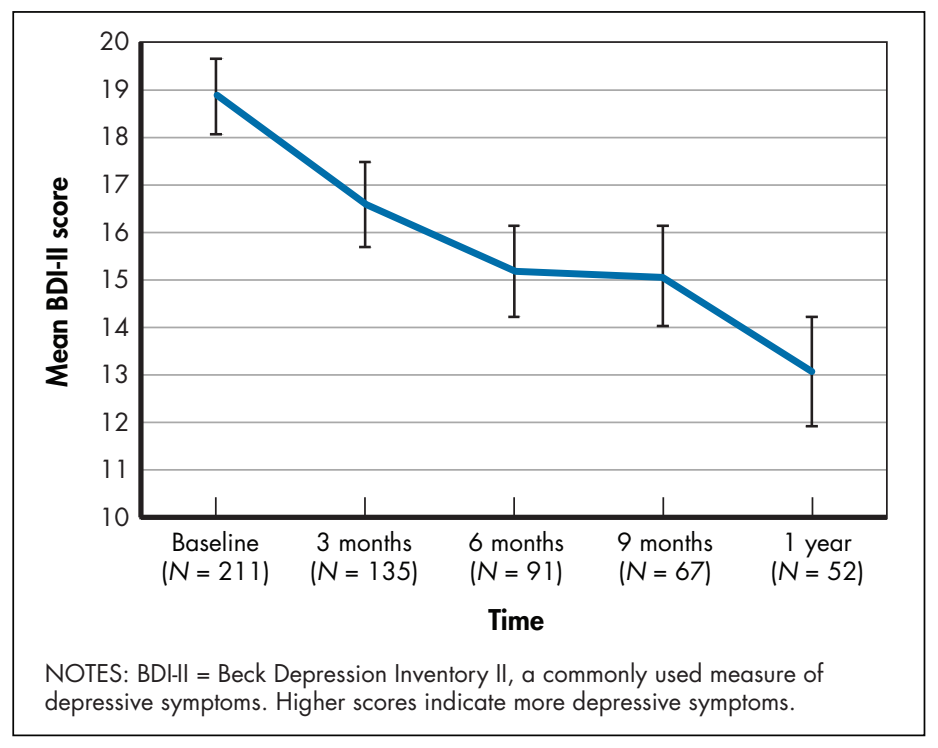

Figure 2. Parent-Reported Child Behavioral Health Symptoms Decreased

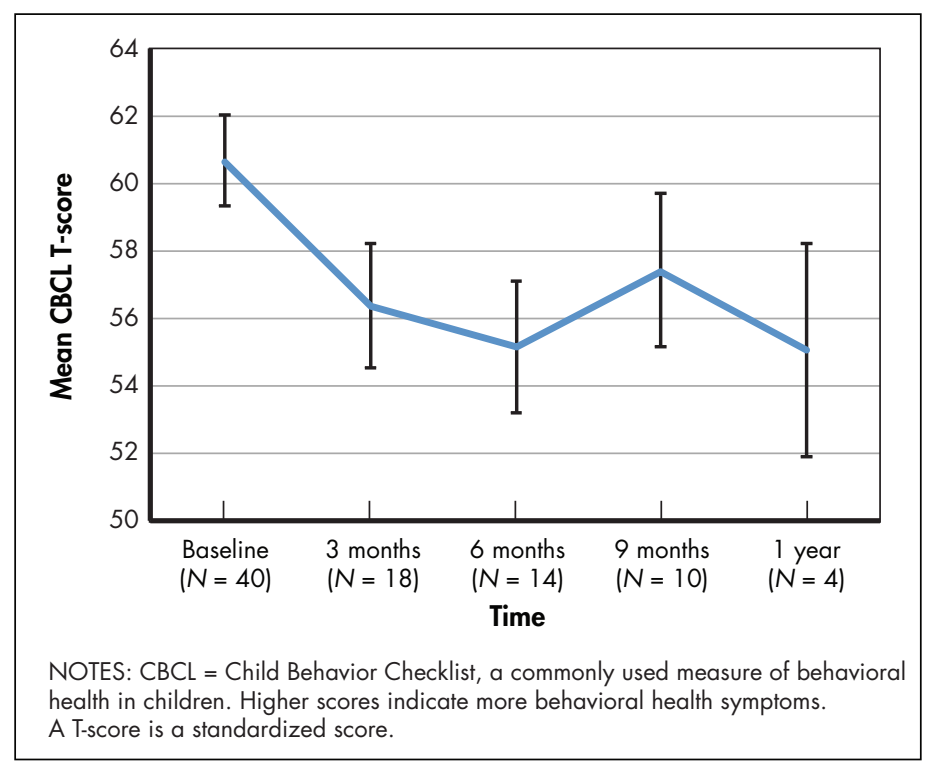

that the care providers were knowledgeable about and sensitive to the experiences of veteran families.

\section{Recommendations}

The RAND evaluation provided recommendations for the UBHC and other providers that wish to learn from or replicate the UBHC model.

\section{Institutionalize and Codify Successful Practices}

The UBHC has established strategies, policies, and procedures to enhance the collaborative effort. However, some of 
these practices have not been institutionalized. For example, there is currently a VA staff member informally acting as a liaison to coordinate care between the two sides of the center; the liaison role could be formalized to ensure that strong communication between organizations continues. More broadly, the VA Clinic at Bay Shore should consider formally protecting the time that their providers spend collaborating.

\section{Facilitate Easier and Closer Collaboration}

Collaboration could be further improved through a series of steps.

Integrate treatment plans. Collaboration would be enhanced by use of integrated treatment plans that staff on both sides of the center contribute to and can readily access.

Share access to patient records. The collaboration would also be enhanced by providers on both sides of the center having easy access to each other's patient records, so that it is easier to track the care a patient is receiving from other providers.

Provide secure email. It would also be helpful if providers could securely email each other, so that communicating about a shared patient does not require a phone call or in-person consultation. This would be particularly important for a larger center, as the informal communication strategies used by the UBHC are difficult to scale up.

\section{Ensure Adequate Capacity (Staffing and Space) to Meet Patient Needs}

If patient interest in the center continues to grow, the UBHC may benefit from expanded staffing and physical space. In particular, increased staff at the VA Clinic at Bay Shore would ensure that there is availability to serve veterans who have family members receiving services on the Feinberg Division side of the UBHC.

\section{Provide a Continuum of Evidence-Based Services}

As more settings work to serve veterans and their families experiencing behavioral health problems, it will be important to deliver evidence-based interventions to address those problems and, additionally, to ensure that needs are met across a continuum of services, including prevention (e.g., psychoeducation) and other types of support (e.g., financial and legal support, family services).

\section{Prioritize Outcome Monitoring and Quality Improvement for the Center as a Whole}

To increase the integration and coordination of services, as well as to enable better tracking of patient outcomes over time, the entire UBHC should implement the same set of patient-reported outcomes measures. This would better inform patient care and enable ongoing quality-improvement efforts across the partnering entities.

\section{Conclusions}

Overall, the UBHC has successfully implemented a promising public-private partnership model for providing, in the same facility, behavioral health care for veterans and their families. Providers coordinated efforts to deliver high-quality care, the center geared up to deliver a range of therapeutic services for a large number of patients in a relatively short time, patients were satisfied with the services they received, and patients' symptoms and functioning improved significantly over time.

The UBHC provides care that is oriented toward the needs of veterans' families. Family members we spoke with expressed that the UBHC plays a vital role in their communities, citing that, in their experience, providers not affiliated with the VA are insensitive to the impact of posttraumatic stress disorder and other special issues facing the families of veterans. Family members saw the UBHC as a unique place where military families could receive care and understanding. UBHC staff and patients alike touted the advantages of coordinated care for the different members of a family.

Although the model has been successfully implemented, with strong preliminary outcomes, there are areas for improvement. Although staff and patients were happy with the collaborative relationships between providers, collaboration could still be closer than it currently is. Staff members regularly have to circumvent various challenges to collaboration.

Other partnerships between local VA centers and private health systems can learn from the UBHC launch and implementation. Some barriers to establishing the center could be circumvented by other programs. For example, building the center was a complicated process, but some of the barriers could potentially be avoided by close communication between the private organization and the appropriate VA staff through all phases of development, with key players at the table from the start. Another potential barrier for other potential partnerships is cost. However, initial expenses could be reduced by using an existing facility rather than building a new one. Further, ongoing expenses could be reduced by billing patients from the start.

Overall, the evaluation suggests that a collaborative model of behavioral health care has been successfully implemented by the UBHC and has great potential to help veterans and their families. 
Support for this work was provided by the New York State Health Foundation (NYSHealth). The mission of NYSHealth is to expand health insurance coverage, increase access to high-quality health care services, and improve public and community health. The views presented here are those of the authors and not necessarily those of NYSHealth or its directors, officers, and staff.

This brief describes work done in RAND Health documented in The Unified Behavioral Health Center for Military Veterans and Their Families: Documenting Structure, Process, and Outcomes of Care, by Nicole K. Eberhart, Michael Stephen Dunbar, Olena Bogdan, Lea Xenakis, Eric R. Pedersen, and Terri Tanielian, RR-1647-NYSHF, 2016 (available at www.rand.org/t/RR1647). To view this brief online, visit www.rand.org/t/RB9938. The RAND Corporation is a research organization that develops solutions to public policy challenges to help make communities throughout the world safer and more secure, healthier and more prosperous. RAND is nonprofit, nonpartisan, and committed to the public interest. RAND's publications do not necessarily reflect the opinions of its research clients and sponsors. RAND ${ }^{\circledast}$ is a registered trademark. ๑ RAND 2016

Limited Print and Electronic Distribution Rights: This document and trademark(s) contained herein are protected by law. This representation of RAND intellectual property is provided for noncommercial use only. Unauthorized posting of this publication online is prohibited. Permission is given to duplicate this document for personal use only, as long as it is unaltered and complete. Permission is required from RAND to reproduce, or reuse in another form, any of our research documents for commercial use. For information on reprint and linking permissions, please visit www.rand.org/pubs/permissions.html. 